\title{
Simulation and Phantom Study of the Acoustic Nonlinear $B / A$ Parameter for Biological Tissues by Using Comparative Method
}

\author{
Jinhua $\mathrm{Yu}^{1,2^{*}}$, Xue $\mathrm{Cai}^{1}$, Yuanyuan Wang ${ }^{1}$, Youneng $\mathrm{Ma}^{1}$ \\ 1 The Department of Electronic Engineering, Fudan University, 220 Handan Road, Physics Building, 212, \\ Shanghai, CN 200433. \\ 2 The Key Lab of Medical Imaging Computing and Computer Assisted Intervention of Shanghai. \\ * Corresponding author. Tel.: (+86) 021-65643202; email: jhyu@fudan.edu.cn \\ Manuscript submitted April 10, 2014; accepted July 20, 2014.
}

doi: 10.17706/ijbbb.2014.4.6.438-447

\begin{abstract}
The nonlinearity parameter $B / A$ is an important indicator of the nonlinear acoustical effects for biological tissues. This paper investigates nonlinear $B / A$ parameter for biological tissues by using comparative method. The investigations are carried out in both computer simulation and phantom study. In simulation study, Pseudo-spectral time domain (PSTD) method for second-order acoustic equation with a novel un-split Perfectly Matched Layer is proposed to improve the simulation efficiency and meanwhile maintain high accuracy. In phantom study, an experimental platform based on the Ritec SNAP-5000 system is designed. Experiments in simulation and phantom study achieve high estimation accuracy for $B / A$ with percentage error less than 3\%. Experimental results show that the comparative method can provide accurate $B / A$ measurement for biological tissues.
\end{abstract}

Key words: Acoustic Nonlinearity, $B / A$ Parameter, Comparative method, Pseudo-spectral Methods.

\section{Introduction}

Ultrasound imaging has been widely used in clinical diagnosis for more than half century. Current ultrasound imaging methods, however, are mostly based on the theory of linear acoustic propagation. In 1980, Muir and Carstensen [1] pointed out that the nonlinear phenomena, which occurred as the ultrasonic wave propagating through the tissues, had to be considered. Starritt et al. [2] even observed the ultrasonic nonlinear phenomena in muscle tissue in 1985. The acoustic nonlinearity draws more and more attention in recent years. Harmonic imaging, which has been provided in most of current commercially available ultrasound machines, is a good example of the imaging that makes use of the acoustic nonlinearity.

After transmitting a wave with a single frequency, the velocity of this wave changes as it propagates through tissues at different depths. The crest velocity is higher than the trough velocity, so the wave becomes distorted. Additional frequency components that are integer multiples of the initial frequency are created. These components are called "harmonic frequencies" [3]. As the wave continues to travel, the infinitesimal amounts of harmonics are created and the transmitting sinusoidal wave becomes more distorted. The nonlinear effects are corresponding to the harmonic creating and accumulating process. The quantification of acoustic nonlinearity is determined by the nonlinear parameter $B / A$, which is an important indicator of harmonic increase in propagating ultrasound.

The parameter $B / A$ is a measure of the nonlinearity of the equation of state. If the propagation of sound is an isentropic process, the general state equation of the pressure for constant entropy can be written as a 
Taylor series:

$$
p=(\partial p / \partial \rho)_{0} \rho+(1 / 2) \cdot\left(\partial^{2} p / \partial \rho^{2}\right)_{0} \rho^{2}+(1 / 3 !) \cdot\left(\partial^{3} p / \partial \rho^{3}\right)_{0} \rho^{3}+\cdots
$$

where $p$ is the pressure field and $\rho_{0}$ is the ambient density. Defining $A=(\rho \cdot \partial P / \partial \rho)_{0}, B=\left(\rho^{2} \cdot \partial^{2} P / \partial \rho^{2}\right)_{0}$, the parameter $B / A$ is a measure of the quadratic term in the pressure-density relation. It has been recognized that the nonlinear parameter $B / A$ can provide more information than linear ones for tissue characterization. In recent years, as a new parameter for ultrasound diagnosis, the application of $B / A$ has a promising prospect in both ultrasound diagnosis and biological acoustics.

There are two groups of methods for $B / A$ measurement: the thermodynamic method and the finite amplitude method [4]. Since thermodynamic method requires rigorous experimental setup, it is not applicable in clinical application. For the methods based on finite amplitude, different approaches utilizing single-frequency transmission or multiple-frequency transmission [5] have been proposed.

The pump wave method, which is proposed by Sato et al. [6] in 1983, is a popular method based on multiple-frequency transmission. This method employs a high-frequency acoustic pump wave propagating perpendicularly to a low-frequency acoustic probe wave. The probe wave is modulated due to the propagation. The $B / A$ parameter is measured by estimation of the phase shift of the probe wave. Since the difficulty and high requirement of phase measurement, this method is not effective.

Another multiple-frequency method is based on the nonlinear generation of different frequency using the parameter array technique [7], [8]. When two high-frequency acoustic waves propagating through a nonlinear medium in the same direction, a low-frequency difference wave, which involves the nonlinear parameter, is produced. The nonlinear parameter can be measured after extracting the difference wave. Generally the difference wave is weak, so it is difficult to extract it.

The methods based on single-frequency transmission can be further divided into direct method [9] and comparative method [10], [11].

After transmitting a wave at single frequency, the pressure amplitude of the second harmonic wave is correlated with nonlinear parameter according to the Fubini solution. Then, the nonlinear parameter can be measured by extracting both the pressure amplitude of the fundamental at the source and the pressure amplitude of the second harmonic components along the propagation axis. Due to the absolute measurement of second harmonic, the result of this method is highly affected by the experiment condition.

To avoid the absolute measurement of second harmonic, Gong et al. [10] proposed an insertion-substitution method. This method stresses on the second harmonic changes as propagating in two different media. One with the known nonlinear parameter is the reference medium, respectively. The second, with the unknown nonlinear parameter, is inserted in the ultrasound field and replaces he reference medium over certain length. When the inserted unknown medium has the same length as the reference medium, the inserted-substitution method can be simplified to comparative method.

Varray et al. [12], [13] investigated the above methods based on finite amplitude and concluded that the comparative method is an optimal choice due to its higher accuracy and experimental simplicity, which also accords with our experimental results.

In this paper, we study the nonlinear $B / A$ parameter for different biological tissues by using comparative method. The study is carried out in both simulation and phantom experiment. In the simulation study, we extend the Pseudo-spectral time domain (PSTD) method to second-order and numerically solve the Westervelt equation. To deal with the simulation truncation problem, a new un-split Perfectly Matched Layer (PML) is also developed for second-order equation. In the phantom study, Ritec SNAP-5000 system (RITEC, Inc. Warwick, RI) is used. The experiment setup and procedure are discussed.

\section{Method}




\subsection{Simulation}

Several approaches are reported to model the nonlinear propagation of acoustic waves in inhomogeneous media. Some of the approaches are based on the widely-used Khokhlov-Zabolotskaya-Kuznetsov (KZK) equation [14]. Pinton et al. [15] proposed a heterogeneous nonlinear attenuating full-wave model and numerically solved the equation by using the finite difference time domain (FDTD) algorithm. Compared with the KZK equation or parabolic equation, the full-wave equation is not limited by the paraxial approximation and thus it provides a more accurate description of an ultrasonic beam in the off-axis region.

In this paper the full-wave Westervelt equation [16] is used which has the form of:

$$
\nabla^{2} p-\left(1 / c_{0}^{2}\right) \cdot\left(\partial^{2} p / \partial t^{2}\right)+\left(\delta / c_{0}^{4}\right) \cdot\left(\partial^{3} p / \partial t^{3}\right)+\left(\beta / \rho_{0} c_{0}^{4}\right) \cdot\left(\partial^{2} p^{2} / \partial t^{2}\right)=0
$$

where $p$ is the pressure field, $c_{0}$ is the propagation speed, $\rho_{0}$ is the ambient density, $\delta$ is the diffusivity of sound, and $\beta$ is the coefficient of nonlinearity $(\beta=1+B / 2 A)$. The first two terms represent the linear wave equation, the third term accounts for thermo viscous diffusivity, followed by nonlinearity. The Westervelt equation can be numerically solved by finite-difference time-domain (FDTD) method. Since FDTD need a huge number of discrete gird to get desirable accuracy, the calculation load and memory requirement are enormous. In this paper, the Fourier Pseudo-spectral (PS) method is used to reduce the computation cost. By using of the properties of the Fourier transform (FT), the partial derivative in time domain and space domain can be calculated. For instance, n-order derivatives in the $\mathrm{x}$-axis can be written as:

$$
\partial^{n} p / \partial x^{n}=F_{x}^{-1}\left\{\left(i k_{x}\right)^{n} F_{x}[p(r)]\right\}
$$

where $k_{x}$ and $F_{x}$ are the angular frequency and FT relates to the $x$ direction, $F^{-1}$ denote inverse FT. The partial derivatives in the $y, z$ axis and the time domain can be calculated in the same way. By using Fourier transform, infinite order of accuracy of derivatives can be acquired. In this way, PSTD method can maintain high calculation accuracy with much less discrete grid, and thus less computation cost than FDTD.

To limit the simulation field into a finite space, PML is used to truncate the outgoing waves. In order to solve the wraparound effect caused by FT, a new PML for second-order equation is developed. Suppose $c_{0}$ is the speed of sound, $\omega$ is angular frequency, $\hat{p}$ is the Fourier transform of the acoustic pressure $p$, and $j$ is the imaginary unit, the PML solution for $x$-axis can be written as:

$$
\left(1 / c_{0}^{2}\right) \cdot(j \omega)^{2} \beta=\left(/ s_{x}\right) \cdot(\partial p / \partial x) \cdot\left(\left(/ s_{x}\right) \cdot(\partial p / \partial x)\right)+\partial^{2} p / \partial y^{2}+\partial^{2} p / \partial z^{2}
$$

where $S_{x}=k_{x}+\sigma_{x} / \alpha_{x}+j \omega$ is the stretching tensor, here $\sigma_{x} \geqslant 0$ is the attenuation factor used for the attenuation of propagating waves, $k_{x} \geqslant 1$ and $\alpha_{x} \geqslant 0$ serve to enhance the attenuation of evanescent and near-grazing waves.

The solutions for other axis are the same way. The details about the implementation of PML can be found in our paper [17]. This algorithm needs no extra storage, and it leads to easier implementation without splitting the pressure field.

\subsection{Comparative Method}

The comparative method (CM) is firstly proposed by Gong et al. in [10]. It is a particular example of the insertion-substitution method. The idea of the insertion-substitution method is to measure the second harmonic change as transmitted wave propagating in two different media. One is a reference medium with known $B / A$ value and length $L$, and the second one, whose $B / A$ value is unknown, is inserted into the reference medium over a length $d$. Suppose the sound velocity and density of these two media are known, then the nonlinear coefficient of the unknown medium can be expressed as, 


$$
\beta_{i} / \beta_{0}=\left[\left(p_{2 i} / p_{20}\right) \cdot\left(1 / D^{\prime} D^{\prime \prime}\right) \cdot(L / d)-L / d+1\right] \cdot\left(\left(\rho c^{3}\right)_{i} /\left(\rho c^{3}\right)_{0}\right) \cdot\left(1 / D^{\prime \prime}\right)
$$

where $\beta, \rho$ and $c$ with the subscript 0 are the density and velocity of the reference medium, and with the subscript $i$ are of the unknown tissue; $p_{2 i}$ and $p_{20}$ are the second harmonic pressure amplitude of unknown medium and reference medium; $D^{\prime}$ and $D^{\prime \prime}$, which can be written as,

$$
\begin{aligned}
& D^{\prime}=2(\rho c)_{0} /(\rho c)_{0}+(\rho c)_{i} \\
& D^{\prime \prime}=2(\rho c)_{i} /(\rho c)_{0}+(\rho c)_{i}
\end{aligned}
$$

are transmission coefficients, respectively.

For high attenuation medium, Gong et al. [11] showed the insertion-substitution method could be improved by considering attenuation coefficient. Then equation (5) becomes,

$$
\beta_{i} / \beta_{0}=\left[\left(p_{2 i} / p_{20}\right) \cdot(L / d) \cdot\left(1 /\left(I_{1} I_{2}\right)\right)-(L / d-1) \cdot\left(I_{2} / I_{1}\right) \cdot\left(D^{\prime} D^{\prime \prime}\right)\right] \cdot\left(\left(\rho c^{3}\right)_{i} /\left(\rho c^{3}\right)_{0}\right) \cdot\left(1 / D^{\prime} D^{\prime 2}\right)
$$

where $I_{1}=\exp \left(-\alpha_{1} d\right)$ and $I_{1}=\exp \left(-\alpha_{2} d / 2\right)$ are related to the attenuation coefficient, $\alpha_{1}$ and $\alpha_{2}$ are the attenuation coefficient of fundamental and harmonic wave, respectively.

If the reference medium and insertion medium has the same length, namely, $L$ equals $d$, then the insertion-substitution method can be simplified as,

$$
\beta_{i} / \beta_{0}=\left[\left(p_{2 i} / p_{20}\right) \cdot\left(1 /\left(I_{1} I_{2}\right)\right)\right]\left(\left(\rho c^{3}\right)_{i} /\left(\rho c^{3}\right)_{0}\right) \cdot\left(1 / D^{\prime} D^{\prime 2}\right)
$$

This formulation indicates the comparative method (CM), which is simpler than the common insertion-substitution method.

As the insertion-substitution method, in comparative method, we also need to assess the second harmonic changes as in two different media with same length. After extracting the second harmonic pressure amplitudes ( $p_{2 i}$ and $p_{20}$, respectively) of the receive signals, we can get the $B / A$ value of the unknown medium.

\subsection{Phantom Experiment Setup}

A diagram of the mechanical setup for phantom experiment is shown in Fig. 1. Ritec SNAP-5000 system (RITEC, Inc. Warwick, RI) is used to generate pulse wave with certain frequency. Signals from nonlinear effects are often several orders of magnitude smaller than the linear signals and can be lost in the noise. The Ritec SNAP-5000 system regarded as the first commercially available state-of-the-art ultrasonic instrument, has high power RF tone burst output and a phase-sensitive superheterodyne receiver. So this system makes the study of nonlinear properties for media more easily and accurately.

Two separate transducers are used with one as the transmitter and the other one as the receiver. The planar transducers with central frequency of $10^{6} \mathrm{~Hz}$ and $2.25 \times 10^{6} \mathrm{~Hz}$ are used (PANAMETRICS V302-SU and V304-SU). Since comparative method is used, water is used as the reference material. The receiving transducer can be immersed into the water. The centrals of the transmitter and the receiver have to be set in line to reduce the interfering. In comparative method, two consecutive measurements of the second-harmonic pressure amplitude are taken, one with the reference and the second with the unknown tissue.

The healthy porcine tissues were studied in our phantom experiment. Since we measured each biological tissue separately, each tissue can be regarded as a homogeneous medium. Suppose the nonlinear coefficients of water and unknown biological sample are $\beta_{0}$ and $\beta_{i}$ respectively, then $\beta_{i}$ can be estimated by comparative method using equation (8). 


\section{Experimental results}

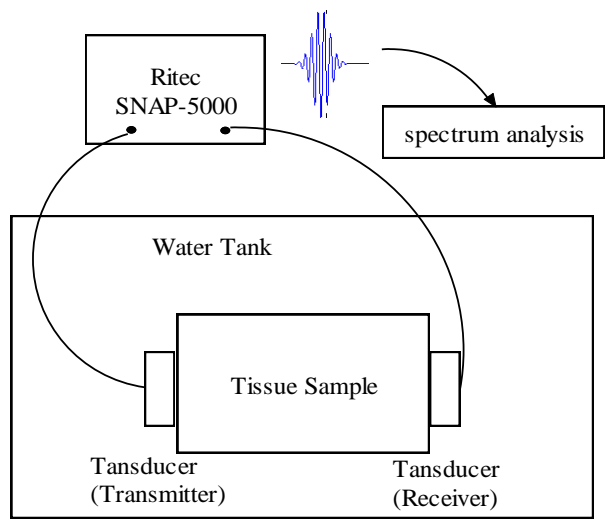

Fig. 1. Diagram of the experiment setup.

In simulation study, a sinusoidal pulse modulated by a Gaussian function is used as the source:

$$
S(t)=-\cos (2 \pi f t) \exp \left(-\left(2 \pi\left(t-t_{0}\right)^{2}\right) / \tau^{2}\right)
$$

where $f=10^{6} \mathrm{~Hz}, t_{0}=0.8 \tau, \tau=3 \times 10^{-6}$. Four biological tissues (water, kidney, liver, muscle) are simulated with water as the reference tissue. The acoustic parameters of simulated tissues which are tunable in the program can be found in references and textbook. The preset $B / A$ values for water, kidney, liver and muscle are 5.2, 7.2, 6.8, and 7.5, respectively. The transducer used in this simulation is a linear probe with 11 elements. The kerf between each element is 2 . Fig. 2 shows an example of the source wave and the receive signal. The transmitting sinusoidal pulse with 10 cycles propagates through the tissues, and then we receive the signal every five millimeters. The receive signals are then sent to spectrum analysis. For comparative method, it is important to measure the second harmonic pressure amplitude. From Fig. 3 we can see that the second harmonic wave is created at the receive signal. To extract the second harmonic pressure amplitude, the receive signals are filtered with a fourth-order Butterworth band-pass filter. The nonlinearity coefficients $\beta$ are then calculated by using (8).

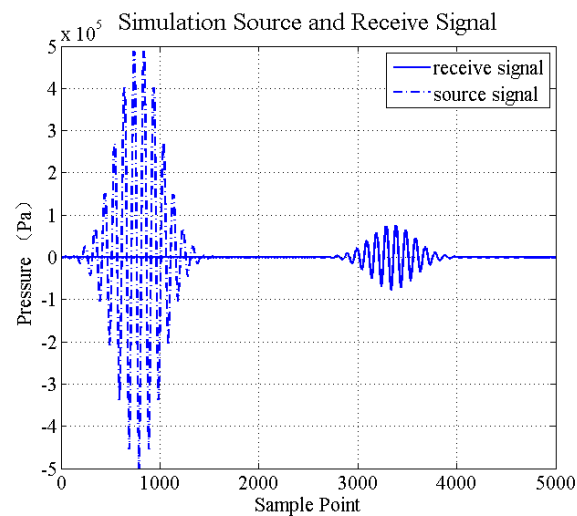

Fig. 2. Illustration of the source wave and received signal in simulation.

Fig. 4 shows the measurement values of nonlinear coefficient for kidney, liver and muscle along the propagation distance. We can see at different distances, the measurements of nonlinear coefficient are almost the same, and are close to the theoretical value. That indicates the accuracy of the CM does not rely on the distance. Fig. 5 shows the measurement errors of nonlinear coefficient for the tissue samples along the different propagation distance. The average error of $B / A$ measurement for all tissues is $2.88 \%$. 

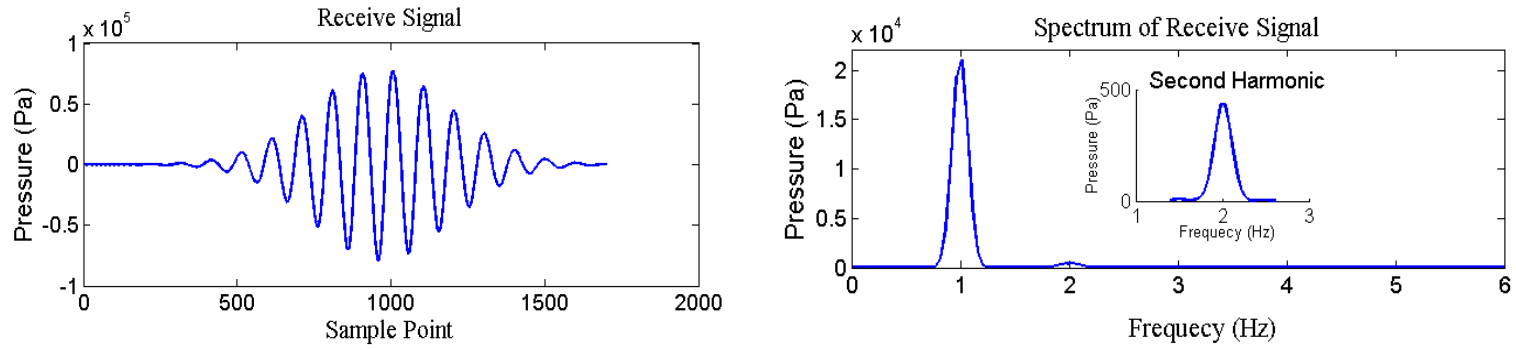

Fig. 3. Illustration of simulation receive signal.

Table 1. Acoustic parameter of tissues

\begin{tabular}{cccccc}
\hline \hline \multirow{2}{*}{ Phantom } & \multirow{2}{*}{$\begin{array}{c}\text { Density } \\
\left(\mathrm{kg} / \mathrm{m}^{3}\right)\end{array}$} & $\begin{array}{c}\text { Velocity } \\
(\mathrm{m} / \mathrm{s})\end{array}$ & \multicolumn{2}{c}{$\begin{array}{c}\text { Attenuation } \\
(\mathrm{dB} / \mathrm{m})\end{array}$} & \begin{tabular}{c} 
Length(1 \\
\cline { 4 - 5 } $\left.0^{-2} \mathrm{~m}\right)$
\end{tabular} \\
\hline kidney & 1050 & 1569 & 0.005 & 0.101 & 1.9 \\
liver & 1068 & 1579 & 0.005 & 0.108 & 1.8 \\
muscle & 1074 & 1615 & 0.007 & 0.130 & 2 \\
\hline \hline
\end{tabular}

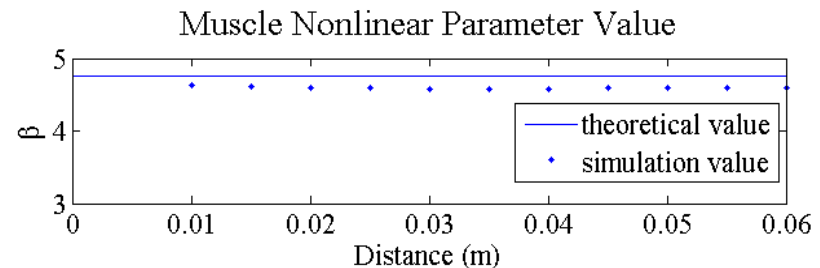

Kidney Nonlinear Parameter Value

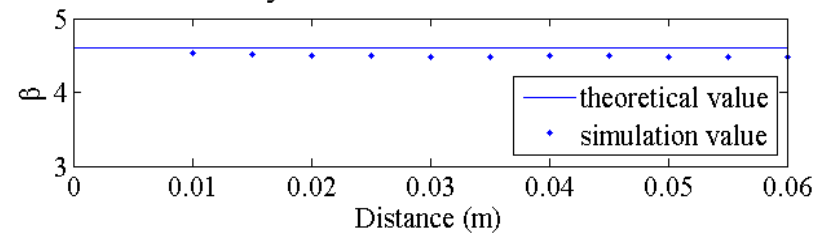

Liver Nonlinear Parameter Value

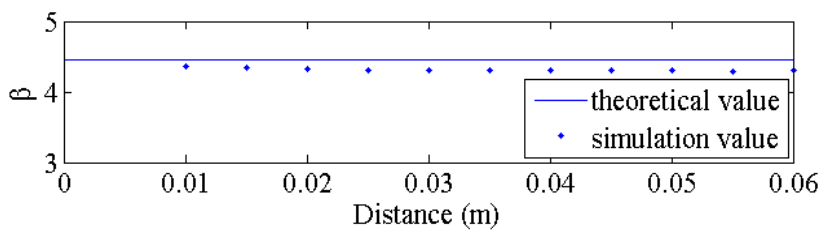

Fig. 4. The illustration of nonlinear coefficient $\beta$ measurement in simulation.

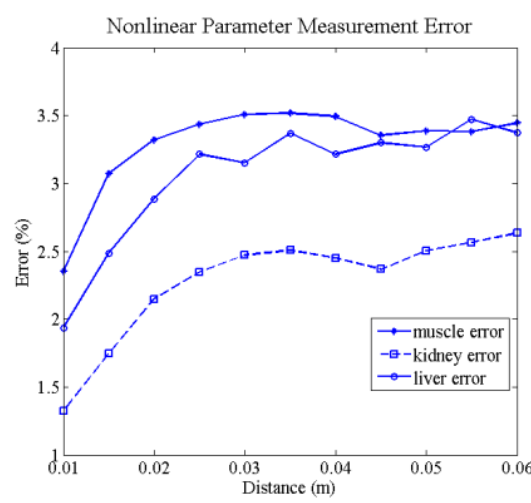

Fig. 5. The illustration of percentage error of $B / A$ measurement in simulation. 


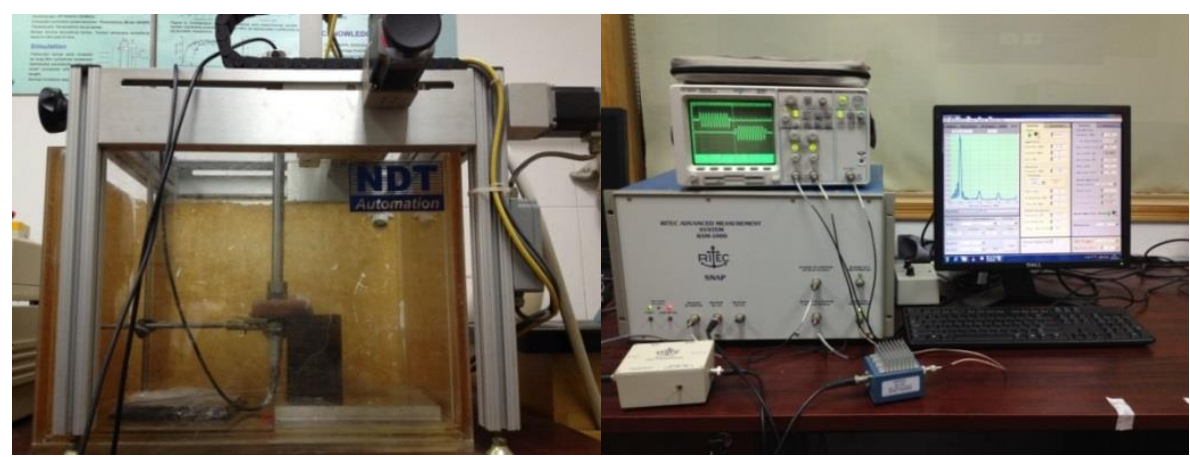

Fig. 6. Mechanical setup of the phantom study.
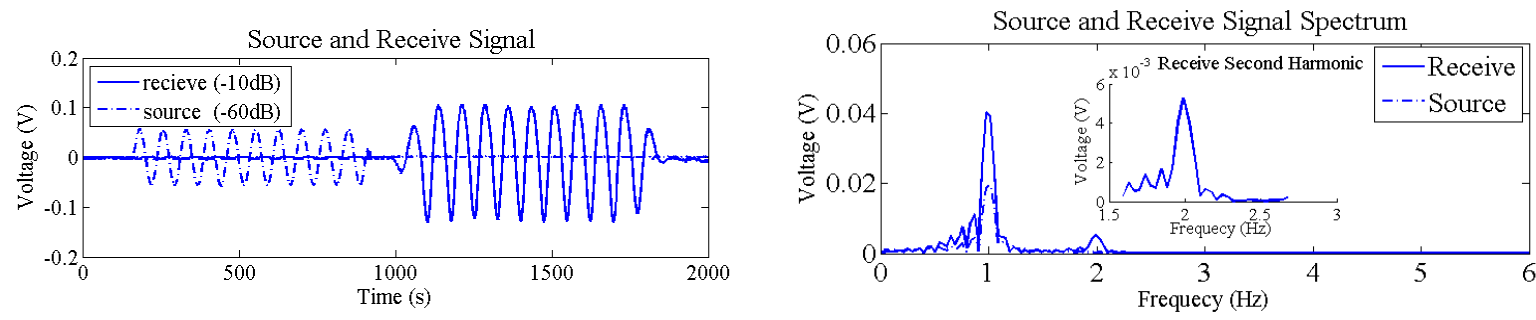

Fig. 7. Illustration of source/receive signal and the corresponding spectrum in phantom study.

Fig. 6 shows the photo of the mechanical setup of our phantom study. Table.1 summarizes the acoustic parameters of the tissues. A gated tone burst generated by the Ritec SNAP-5000 system is used to excite the transducer. The gated tone burst is 10 cycles and the repetition ferequency $50 \mathrm{~Hz}$. Fig. 7 shows the source wave and received signals. In Fig. 7 the source wave was attenuated $60 \mathrm{~dB}$ and received signal was attenuated $10 \mathrm{~dB}$.

In our phantom experiment, the measurement values of $B / A$ parameter of kidney, liver and muscle are 7.4, 6.6, 7.6, which are close to the values in references and textbook. The average estimation error of $B / A$ in phantom study is $2.38 \%$. That shows comparative method also has high accuracy in phantom experiment.

In ultrasonic diagnosis and tissue characterization, $B / A$ imaging is more useful than $B / A$ measurement. However, the $B / A$ imaging is rely on the accuracy of the $B / A$ measurement. From the simulation and phantom study, we can see that the comparative method can provide accurate $B / A$ measurement for biological tissues. Since measurement of $B / A$ by CM can only provide average $B / A$ value of tissues, $B / A$ imaging should be implemented to confirm the accuracy of this measurement.

For inhomogeneous tissues, the $\beta_{i}$ along the propagation distance can be estimated by [12]:

$$
\int_{0}^{x} \beta^{\prime}(u) d u=\left(p_{2 i}(x) / p_{20}(x)\right) \cdot\left(\left(\rho c^{3}\right)_{i} /\left(\rho c^{3}\right)_{0}\right) \cdot \beta_{0} I_{0}(x)
$$

where $\beta^{\prime}(u)=\beta_{i}(u) \exp \left(\int_{0}^{u}-2 \alpha_{1 i}(v) d V-\int_{u}^{x} \alpha_{2 i}(v) d V\right), I_{0}(\mathrm{x})=\left(\exp \left(-2 \alpha_{10} x\right)-\exp \left(-\alpha_{20} x\right)\right) /\left(\alpha_{20}-2 \alpha_{10}\right)$, $\alpha_{10}, \alpha_{20}$ are the attenuation coefficient of fundamental and harmonic wave for reference medium, and $\alpha_{1 i}$, $\alpha_{2 i}$ are the attenuation coefficient of fundamental and harmonic wave for unknown medium. At the fixed angle $\theta$, suppose the projection data $p(u, \theta)$ is:

$$
p(u, \theta)=\left(p_{2 i}(x) / p_{20}(x)\right) \cdot\left(\left(\rho c^{3}\right)_{i} /\left(\rho c^{3}\right)_{0}\right) \cdot \beta_{0} I_{0}(x)
$$

then the distribution of $\beta^{\prime}(u)$ can be reconstructed by using filtered back projection (FBP). After 
multiplying the attenuation term, the $B / A$ tomography imaging can be obtained [18].

Fig. 8 shows the $B / A$ imaging for a simple phantom model in simulation environment. This phantom is composed of a background with $\beta$ value of 3.5 and rectangle with $\beta$ value of 7 in the middle (see Fig. 8 (a)). The region between the two red lines delineates the reference medium which is necessary in CM. Fig. 8(b) shows the FBP reconstructed imaging, the different $\beta$ structures of the set phantom can be observed. And from Fig. 8(c) we can see the distribution of the $\beta$ value along $x$ direction is in reasonable accordance with the set value.

All the experiments show that the comparative method is a potential approach to estimate $B / A$ value for biological tissues.

(a)

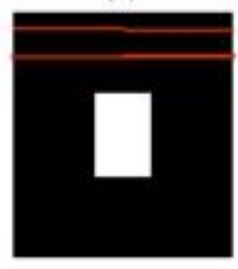

(b)

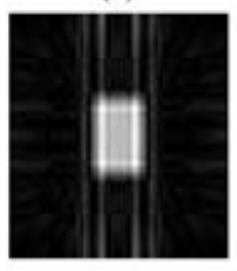

(c)

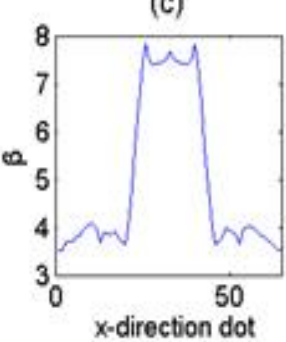

Fig. 8. $B / A$ tomography result: (a) tissue model, (b) $B / A$ tomography result, (c) $\beta$ distribution along $X$ direction in (b).

\section{Discussion and Conclusion}

The nonlinear parameter describes the distortion of finite amplitude as it propagates through the medium. The nonlinear parameter varies in different tissues and can provide more information on structural features and pathological state of tissues. The measurement of nonlinear parameter is the basement of an ultrasound novel parameter imaging for biological tissue characterization.

Several methods based on finite amplitude theory can determine the nonlinear parameter in tissues. Each method has its advantage and disadvantage. Comparing several classical finite amplitude methods in simulation environment, Varry et al.[13] point out that comparative method has higher accuracy and simpler to experiment operate.

We investigate nonlinear $B / A$ parameter for biological tissues by using comparative method in both computer simulation and phantom study. Experimental results show that the comparative method can provide accurate $B / A$ measurement for biological tissues. Additionally, $B / A$ imaging by using the comparative method combined with FBP is acceptable, since it can get the right distribution of $B / A$ for a simple tissue. However, such transmission mode ultrasound imaging is not suitable for clinical ultrasound medical exam. The projection data collection for image reconstruction consumes time and needs large storage.

Considering the clinical suitable, several efforts have been made to simplify the $B / A$ imaging. Akiyama and Fujii et al. [19] proposed a method to measure the $B / A$ parameter in echo mode signals by transmitting pulse of center frequency $f_{0}$ and $2 f_{0}$. They supposed that the acoustical scattering characteristics are the same in the second harmonic wave and in a wave transmitted at double frequency. The echo amplitude of the second harmonic component for a transmission at frequency $f_{0}$ and the echo amplitude of the fundamental component for a transmission at frequency $2 f_{0}$ were compared to suppress the scatterers' impacts. And from the ratio of these two echo amplitudes the $B / A$ in different propagation depth was derived. This method is an advancement of $B / A$ imaging in echo mode and is relatively easy to implement, but it has several limitations. Varry et al. [13] [20] implemented the comparative method to obtain $B / A$ 
imaging in echo mode. They supposed that, with the fully develop speckle, the RF image is related to its pressure field if the impact of the scattering is successfully suppressed. Filtering in this method is important to the result, and the filtered RF signal may distort. Such distortion may lead a bad impact in $B / A$ imaging.

Up to now, some of the existing $B / A$ measurements, especially the comparative method, can provide relatively high accuracy. But in terms of $B / A$ imaging, all existing methods have their own limitations. A nonlinear propagation theoretical background needs to be more specifically understood. The influence of scattering in RF signals needs to be suppressed more efficiently to improve the $B / A$ imaging quality.

\section{References}

[1] Muir, T. G., \& Carstensen, E. L. (1980). Prediction of nonlinear acoustic effects at biomedical frequencies and intensities. Ultrasound in Medicine \& Biology, 6(4), 345-357.

[2] Starritt, H. C., Perkins, M. A., Duck, F. A., \& Humphrey, V. F. (1985). Evidence for ultrasonic finite-amplitude distortion in muscle using medical equipment. Journal of the Acoustical Society of America, 77(1), 302-306.

[3] Choudhry, S., Gorman, B., \& Charboneau, W. (2000). Comparison of tissue harmonic imaging with conventional US in abdominal disease. Radio Graphics, 20(4), 1127-1135.

[4] BjØrnØ, L. (2002). Forty years of nonlinear ultrasound. Ultrasonics, 40(1-8), 11-17.

[5] Fenlon, F. H. (1972). An extension of the Bessel-Fubini series for a multiple-frequency CW acoustic source of finite amplitude. Journal of the Acoustical Society of America, 51(1), 284-289.

[6] Ichida, N., Sato, T., \& Linzer, M. (1983). Imaging the nonlinear ultrasonic parameter of a medium. Ultrasonic Imaging, 5, 295-299.

[7] Nakagawa, Y., Nakagawa, M., Yoneyama, M., \& Kikuchi, M. (1984). Nonlinear parameter imaging computed tomography by parametric acoustic array. Proceeding of the IEEE 1984 Ultrasonics Symposium (pp. 673-676). USA: Dallas, Texas.

[8] Nakagawa, Y., Hou, W., Cai, A., Arnold, N., \& Wade, G. (1986). Nonlinear parameter imaging with finite amplitude sound waves. Proceeding of the IEEE 1984 Ultrasonics Symposium (pp. 901-904). USA: Dallas, Texas.

[9] BjØrnØ, L. (1986). Characterization of biological media by means of their non-linearity. Ultrasonics, 24(5), 254-259.

[10] Gong, X., Feng, R., Zhu, C., \& Shi, T. (1984). Ultrasonic investigation of the nonlinearity parameter $B / A$ in biological media. Journal of the Acoustical Society of America, 76(3), 949-950.

[11] Zhang, D., \& Gong, X. (1999). Experimental investigation of the acoustic nonlinearity parameter tomography for excised pathological biological tissue. Ultrasound in Medicine \& Biology, 25(4), 593-599.

[12] Varray, F., Pasovic, M., Cachard, C., Tortoli, P., \& Basset, O. (2009). Acoustic nonlinearity parameter of tissue on echo mode: review and evaluation of the different approaches for $B / A$ imaging. Proceeding of the IEEE 2009 International Ultrasonics Symposium (pp. 41-44). Italy: Rome.

[13] Varray, F., Basset, O., Tortoli, P., \& Cachard, C. (2011). Extensions of nonlinear B/A parameter imaging methods for echo mode. IEEE Transactions on Ultrasonics, Ferroelectrics and Frequency Control, 58(6), 1232-1244.

[14] Fan, T., Liu, Z., \& Tang, M. (2013). Comparative study of lesions created by high-intensity focused ultrasound using sequential discrete and continuous scanning strategies. IEEE Transactions on Biomedical Engineering, 60(3), 763-769.

[15] Pinton, G. F., Dahl, J., Rosenzweig, S., \& Trahey, G. E. (2009). A heterogeneous nonlinear attenuating full-wave model of ultrasound. IEEE Transactions on Ultrasonics, Ferroelectrics and Frequency Control, 56(3), 474-488. 
[16] Jing, Y., Wang, T., \& Clement, G. T. (2012). A k-space method for moderately nonlinear wave propagation. IEEE Transactions on Ultrasonics, Ferroelectrics and Frequency Control, 59(8), 1664-1673.

[17] Ma, Y., Yu, J., \& Wang, Y. (2014). An efficient complex-frequency shifted PML for second-order acoustic wave equation. International Journal for Numerical Methods in Engineering, 97(2), 130-148.

[18] Zhang, D., Gong, X., \& Ye, S. (1996). Acoustic nonlinearity parameter tomography for biological specimens via measurements of the second harmonics. Journal of the Acoustical Society of America, 99(4), 2397-2402.

[19] Fujii, Y., Taniguchi, N., \& Akiyama, I. (2004). A new system for in vivo assessment of the degree of nonlinear generation using the second harmonic component in echo signals. Ultrasound in Medicine \& Biology, 30(11), 1511-1516.

[20] Toulemonde, M., Varry, F., Tortoli, P., Cachard, C., \& Basset, O. (2012). Speckle reduction with multitaper approach to improve B/A imaging. Proceeding of the Acoustics 2012 Nantes Conference (pp. 23-27). France: Nantes.

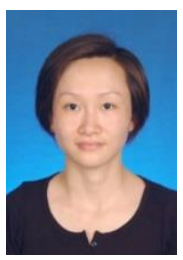

Jinhua Yu received the Ph.D. degree from Fudan University, Shanghai, China, in 2008. She was a postdoctoral fellow at the University of Missouri, USA from 2008 to 2010. She is currently an associated researcher of Fudan University. Her current research interests include medical image analysis and ultrasound imaging.

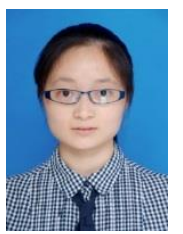

Xue Cai was born in Zunyi City, Guizhou Province, China in 1989. She received the B.S. degree from Sichuan University, Chengdu City, China, in 2012. She is currently pursuing the M.S. degree in electronic engineering in Fudan University, Shanghai, China. Her research interests include ultrasound imaging.

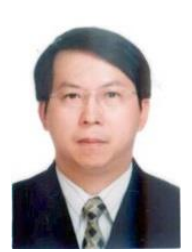

Yuanyuan Wang received the B.Sc., M.Sc. and Ph.D. degrees in electronic engineering from Fudan University, Shanghai, China, in 1990, 1992 and 1994, respectively. During 1994 to 1996, he was a postdoctoral research fellow with the School of Electronic Engineering and Computer Science at University of Wales, Bangor, UK. In May 1996, he went back to Department of Electronic Engineering at Fudan University as an associate professor. He was then promoted to a full professor in May, 1998. He is currently the director of Biomedical Engineering Center and vice dean of Information Science and Engineering School at Fudan University. He is also the author or coauthor of 6 books and 400 research papers. His research interests include medical ultrasound techniques and medical signal processing.

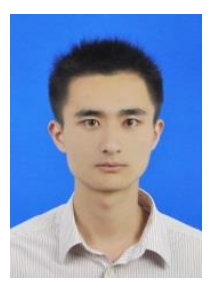

Youneng Ma was born in Zhuzhou City, Hunan Province, China in 1988. He received the B.S. degree from Chongqing University, Chongqing City, China, in 2011. He is currently pursuing the M.S. degree in electronic engineering in Fudan University, Shanghai, China. His research interests include partial fraction expansion and ultrasound imaging. 